\title{
Plasma Biomarkers of Neuropathogenesis in Hospitalized Patients With COVID-19 and Those With Postacute Sequelae of SARS-CoV-2 Infection
}

Barbara A. Hanson, BA, Lavanya Visvabharathy, PhD, Sareen T. Ali, BA, Anthony K. Kang, BS, Tulsi R. Patel, BA, Jeffrey R. Clark, BA, Patrick H. Lim, MS, Zachary S. Orban, BS, Soyoon S. Hwang, PhD, Dawn Mattoon, PhD, Ayush Batra, MD, Eric M. Liotta, MD, MS, and Igor J. Koralnik, MD

Neurol Neuroimmunol Neuroinflamm 2022;9:e1151. doi:10.1212/NXI.0000000000001151

\section{Abstract}

\section{Background and Objectives}

Although patients hospitalized with COVID-19 frequently present with encephalopathy, those with mild initial COVID-19 disease who never required hospitalization also often develop neurologic symptoms as part of postacute sequelae of severe acute respiratory coronavirus type 2 (SARS-CoV-2) infection (neuro-PASC). The pathogenic mechanisms of COVID-19 encephalopathy and neuro-PASC are unknown. We sought to establish biochemical evidence of CNS injury in those patients and their association with neuropsychiatric manifestations and SARS-CoV-2 antigenemia.

\section{Methods}

We recruited hospitalized, posthospitalized, and nonhospitalized patients with confirmed diagnosis of COVID-19 with neurologic symptoms in addition to healthy control (HC) subjects. Plasma neurofilament light chain (pNfL), plasma glial fibrillary acidic protein (pGFAP), and plasma SARS-CoV-2 Nucleocapsid antigen ( $\mathrm{pN} \mathrm{Ag}$ ) were measured by HD-X Simoa analyzer (Quanterix) and compared with neuropsychiatric symptoms, patient-reported quality-of-life measures, and standardized cognitive assessments. Neuroglial scores (pGFAP/pNfL) were calculated to estimate the relative contribution of astroglial and neuronal involvement.

\section{Results}

We enrolled a total of 64 study participants, including 9 hospitalized patients with COVID-19 encephalopathy (CE), 9 posthospitalization neuro-PASC (PNP) patients, 38 nonhospitalized neuro-PASC (NNP) patients, and $8 \mathrm{HC}$ subjects. Patients with CE were older, had higher pNfL and pGFAP concentrations, and more frequent $\mathrm{pN}$ Ag detection than all neuro-PASC groups. PNP and NNP patients exhibited similar PASC symptoms, decreased quality-of-life measures, and cognitive dysfunction, and 1 of the $38(2.6 \%)$ NNP patients had $\mathrm{pN} \mathrm{Ag} \mathrm{detectable} 3$ weeks postsymptoms onset. Patients with neuro-PASC presenting with anxiety/depression had higher neuroglial scores, which were correlated with increased anxiety on quality-of-life measures.

\section{Discussion}

pNfL, pGFAP, and pN Ag measurements indicate neuronal dysfunction and systemic involvement in hospitalized COVID-19 patients with encephalopathy. Detection of SARS-CoV-2 $\mathrm{N} \mathrm{Ag}$ in blood 3 weeks after symptoms onset in a nonhospitalized patient suggests that prolonged antigenic stimulation, or possibly latent infection, may occur. Anxiety was associated with evidence of astroglial activation in patients with neuro-PASC. These data shed new light on SARS-Cov-2 neuropathogenesis and demonstrate the value of plasma biomarkers across the COVID-19 disease spectrum.

\author{
Correspondence \\ Dr. Koralnik \\ igor.koralnik@northwestern.edu
}

\section{MORE ONLINE}

\section{COVID-19 Resources}

For the latest articles, invited commentaries, and blogs from physicians around the world

NPub.org/COVID19

From the Ken and Ruth Davee Department of Neurology (B.A.H., L.V., S.T.A., A.K.K., T.R.P., J.R.C., P.H.L., Z.S.O., A.B., E.M.L., I.J.K.), Feinberg School of Medicine, Northwestern University; Rush Medical College (B.A.H.), Chicago IL; and Quanterix Corporation (S.S.H., D.M.), Billerica, MA.

Go to Neurology.org/NN for full disclosures. Funding information is provided at the end of the article.

The Article Processing Charge was funded by the authors.

This is an open access article distributed under the terms of the Creative Commons Attribution-NonCommercial-NoDerivatives License 4.0 (CC BY-NC-ND), which permits downloading and sharing the work provided it is properly cited. The work cannot be changed in any way or used commercially without permission from the journal. 


\section{Glossary}

CAT = computer adaptive testing; CE = COVID-19 encephalopathy; GLT-1 = glutamate transporter 1; HC = healthy control; MS = multiple sclerosis; NMOSD = neuromyelitis optica spectrum disorder; NNP = nonhospitalized neuro-PASC; PASC = Postacute sequelae of SARS-CoV-2 infection; pGFAP = plasma glial fibrillary acidic protein; $\mathbf{p N f L}=$ Plasma neurofilament light chain; PNP = posthospitalization neuro-PASC; PROMIS = Patient-Reported Outcome Measurement Information System; RT-PCR = reverse transcriptase PCR; SARS-CoV-2 = severe acute respiratory coronavirus type 2.

According to the World Health Organization, more than 260 million people have been diagnosed with severe acute respiratory coronavirus type 2 (SARS-CoV-2) infection, the virus that causes COVID-19, leading to close to 5.2 million deaths between December 2019 and November 26, 2021. ${ }^{1}$ Infections continue to grow despite the availability of vaccines that effectively prevent severe disease and death in most cases. Although acute infection primarily targets the respiratory tract, extrarespiratory organs, including the CNS, are also affected. $^{2,3}$ This results in neurologic symptoms, including encephalopathy, despite the fact that conclusive evidence of active viral replication in the CNS is lacking.

Several studies have investigated blood-based biomarkers of CNS injury in the acute phase of both severe and mild-tomoderate COVID-19 to assess the nature of CNS injury in acute infection. Plasma neurofilament light chain (pNfL) is an intra-axonal structural protein that has been validated as a biomarker for neuroaxonal damage, and plasma glial fibrillary acidic protein (pGFAP) is an astrocytic cytoskeletal protein that is upregulated in activated astrocytes. ${ }^{4,5}$ Both markers have been shown to increase in the acute phase of severe and mild-to-moderate COVID-19, indicating that CNS injury associated with neuronal damage and astrocytic activation occurs with acute infection. ${ }^{6,7}$

A subset of patients with COVID-19 experiences chronic cardiopulmonary, gastrointestinal, and/or neurologic symptoms after the acute phase. These postacute sequelae of SARS-CoV-2 infection (PASC) are observed in patients who experienced severe disease requiring hospitalization and those who had mild and even asymptomatic acute disease. ${ }^{8}$ PASC patients with neurologic symptoms (neuroPASC) most frequently present with cognitive difficulties, headaches, dizziness, disorders of smell and taste, and neuropsychiatric complaints such as new-onset depression and anxiety. ${ }^{9,10}$ The chronic nature of these complaints suggests that ongoing neuroinflammation and/or direct damage to the CNS may persist long after acute infection resolves.

We sought to assess biochemical evidence of CNS injury in acutely encephalopathic COVID-19 patients and neuroPASC patients with chronic neurologic symptoms 1-13 months after either severe acute disease requiring hospitalization or mild initial infection. We then determined the relationship between these biomarkers and acute disease severity and whether they associated with findings of neurologic, cognitive, or neuropsychiatric symptoms.

\section{Methods}

\section{Participants and Study Design}

We recruited patients with COVID-19 who were currently hospitalized with COVID-19 encephalopathy (CE), posthospitalization neuro-PASC (PNP) patients hospitalized for COVID-19 pneumonia, or nonhospitalized neuro-PASC (NNP) patients with mild initial infection between March 2020 and December 2020. Plasma samples and quality of life and NIH Toolbox data were obtained between August 2020 and March 2021. All neuro-PASC patients exhibited neurologic symptoms persisting at least 6 weeks from symptom onset. COVID-19 diagnosis was performed by SARS-CoV-2 reverse transcriptase PCR (RT-PCR) of nasopharyngeal swab and/or SARS-CoV-2 antibody testing. Healthy control samples were obtained from individuals who showed negative results for RT-PCR and serology tests and had no clinical suspicion of infection during the SARS-CoV-2 pandemic. All consenting eligible patients who presented to the NeuroCOVID-19 clinic were included in the study.

\section{Standard Protocols, Approvals, Registration, and Patient Consents}

The study was approved by the Northwestern University Institutional Review Board (STU00212583). All participants were enrolled after giving their written informed consent.

\section{Procedures}

Encephalopathy in hospitalized patients was diagnosed by an attending physician. All patients with neuro-PASC were evaluated by an attending neurologist in the Northwestern University Medical Center Neuro-COVID-19 clinic. PatientReported Outcome Measurement Information System (PROMIS) Computer Adaptive Testing (CAT) assessment of quality of life in cognition (PROMIS CAT v2.0), fatigue (PROMIS CAT v1.0), anxiety (PROMIS CAT v1.0), depression (PROMIS CAT v1.0), and sleep disturbance (PROMIS CAT v1.0) were performed in the outpatient clinic. ${ }^{11,12}$ Cognitive function evaluation was performed using the NIH Toolbox v2.1 instrument, which assessed processing speed (pattern comparison processing speed test); attention (inhibitory control and attention test); executive function (dimensional change card sort test); and working memory (list sort working memory test). ${ }^{13-16}$ All tests were administered by 
Table 1 Study Subject Demographics and Plasma Antigen Concentrations

\begin{tabular}{|c|c|c|c|c|c|c|}
\hline & CE $(n=9)$ & $\operatorname{PNP}(n=9)$ & NNP $(n=38)$ & $\mathrm{HC}(n=8)$ & $p$, all groups & p, CE excluded \\
\hline Age (mean [1 SD]) & $74.9(15.8)$ & $54.1(20.3)$ & $45.5(12.1)$ & 38.5 (10.9) & $<0.001$ & 0.07 \\
\hline Female, n (\%) & $6(66.6)$ & $3(33.3)$ & $26(66.6)$ & $5(62.5)$ & 0.31 & 0.18 \\
\hline Male, n (\%) & $3(33.3)$ & $6(66.6)$ & $13(33.3)$ & $3(37.5)$ & & \\
\hline \multicolumn{7}{|l|}{ Race, n (\%) } \\
\hline White & $6(66.6)$ & $6(66.6)$ & $33(84.6)$ & $6(75.0)$ & & \\
\hline Black & $2(22.2)$ & $1(11.1)$ & $1(2.6)$ & & & \\
\hline Asian & & $1(11.1)$ & $4(10.3)$ & $2(25.0)$ & & \\
\hline Other & $1(11.1)$ & $1(11.1)$ & $1(2.6)$ & & & \\
\hline \multicolumn{7}{|l|}{ Ethnicity, n (\%) } \\
\hline Hispanic & $1(11.1)$ & $2(22.2)$ & $5(12.8)$ & $1(12.5)$ & & \\
\hline Non-Hispanic & $8(88.8)$ & $7(77.7)$ & $33(84.6)$ & $7(87.5)$ & & \\
\hline DPO (median [IQR]) & 20 [10.5-32] & 237 [204-294] & 196 [147-323] & $N A$ & $<0.001$ & 0.96 \\
\hline \multicolumn{7}{|c|}{ Plasma antigen concentration $(\mathrm{pg} / \mathrm{mL})$} \\
\hline \multicolumn{7}{|l|}{ pNfL (median [IQR]) } \\
\hline$<50$ & 186.3 & $5.1[3.0-7.4]$ & $5.4[3.4-7.2]$ & $5.4[3.3-5.8]$ & NA & 0.83 \\
\hline$>50$ & 75.6 [70.6-269.7] & $14.0[6.1-16.7]$ & $8.11[6.7-11.0]$ & 9.25 & $0.03^{a}$ & $0.44^{a}$ \\
\hline \multicolumn{7}{|l|}{ pGFAP (median [IQR]) } \\
\hline$<50$ & 73.6 & $41.0[11.9-68.6]$ & $55.3[42.6-72.0]$ & $40.1[30.5-96.7]$ & NA & 0.80 \\
\hline$>50$ & $198.9[126.3-457.1]$ & 62.4 [23.9-127.7] & $67.5[35.0-91.7]$ & 104.66 & $0.003^{a}$ & $0.97^{\mathrm{a}}$ \\
\hline N-Ag-positive, n (\%) & $5(55.6)$ & & $1(2.6)$ & & & \\
\hline N-Ag (median [IQR]) & $109.5[3.9-4,025]$ & & 0.37 & & & \\
\hline \multicolumn{7}{|c|}{ Neuroglial score (mean [1 SD]) } \\
\hline$<50$ & 0.4 & $6.61(3.20)$ & $12.77(6.34)$ & $11.76(7.15)$ & NA & 0.17 \\
\hline$>50$ & 3.51 (3.57) & $5.69(2.79)$ & $7.89(4.94)$ & 11.32 & $0.07^{a}$ & $0.36^{\mathrm{a}}$ \\
\hline
\end{tabular}

Abbreviations: $C E=$ COVID in-patients with encephalopathy; PNP = posthospitalization neuro-PASC; NNP = never hospitalized neuro-PASC; HC = healthy control; DPO = sample days postonset; pGFAP = plasma glial fibrillary acidic protein; $\mathrm{pNfL}$ = plasma neurofilament light chain.

Bold values indicate any $p$ values $<0.05$ and were considered significant.

${ }^{a} \mathrm{HC}$ excluded.

clinical or research staff in the Neuro-COVID-19 clinic. Both PROMIS and NIH Toolbox results are expressed as T scores with a score of 50 representing the normative mean/median for the US reference population with an SD of 10 . The derivation cohort for PROMIS was selected to represent the demographic composition of the US population. NIH Toolbox T scores are adjusted for age, education, sex, and race/ethnicity. Lower cognition, processing speed, attention, executive function, and working memory $\mathrm{T}$ scores represent worse performance, whereas higher fatigue, anxiety, depression, and sleep disturbance $\mathrm{T}$ scores indicate worse outcomes.

Heparinized plasma samples were obtained at the date of enrollment, concurrent with quality-of-life and cognition measures evaluation for PNP and NNP patients. pGFAP and
pNfL and Nucleocapsid antigen titers were performed by Quanterix Corporation (Billerica, MA) on a Simoa HD-X analyzer using GFAP discovery (cat. 502656) and Nf-Light kits (cat. 502296), respectively. All samples were run in duplicate with the exception of 3 samples with insufficient volume, which were run in singleton. The median coefficient of variation for duplicate measures was $5 \%$ for both pGFAP and $\mathrm{pNfL}$ and was normalized to dilution factor, when appropriate.

\section{Statistical Analysis}

Data were summarized as number of patients (percentage/ frequency), normally distributed variables as mean (SD), or median (interquartile range) for non-normally distributed variables. Group differences for normally distributed data 
A

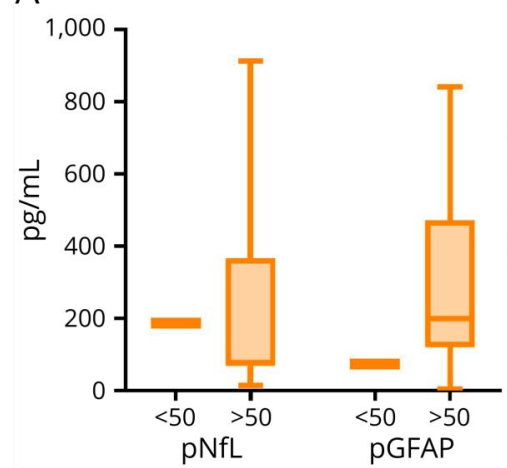

C

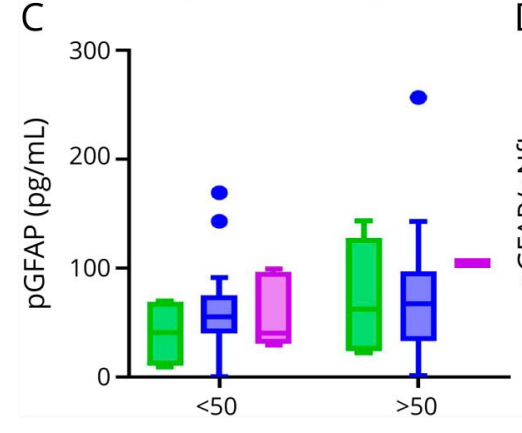

B

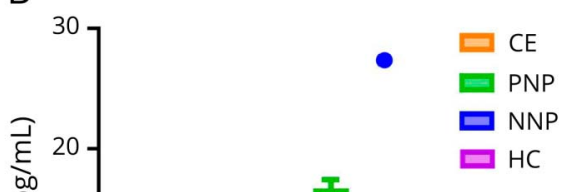

Plasma neurofilament ( $\mathrm{pNfL}$ ) and plasma glial fibrillary acidic protein (pGFAP) concentration stratified by age in (A) patients hospitalized with Covid-19 encephalopathy (CE: younger than 50 years, $n=1$, older than 50 years, $n=8)$ and ( $B$ and $C$ ) patients who experienced Covid-19 pneumonia and are now posthospitalization with neuro-PASC (PNP: younger than 50 years, $n=4$, older than 50 years, $n=5$ ), nonhospitalized neuro-PASC patients (NNP group: younger than 50 years, $n=20$, older than 50 years, $n=18$ ), and healthy control subjects (HC: younger than 50 years, $n=7$, older than 50 years, $n=1$ ). Both pNFL and pGFAP levels are significantly higher in CE older than 50 years than those in all other groups including those older than 50 years (Kruskal-Wallis test; $\mathrm{pNfL}: \mathrm{H}=16.23, p=0.0003$; pGFAP: $\mathrm{H}=7.34, p=0.02$ ). pGFAP/pNfL ratio (D) represents neuroglial score, where higher scores indicate predominance of astrocytic activation and lower scores predominant neuroaxonal damage. were assessed using ANOVA and non-normally distributed data with the Kruskall-Wallis test or the Mann-Whitney test in the case of 2 group comparisons; quantitative data were compared with the Fisher exact test. Pairwise comparisons for normally distributed data were assessed by 2-tailed $t$ tests for independent means, followed by Hedges' $\mathrm{g}$ effect size for $t$ test. Associations between quantitative variables were assessed using the Pearson correlation test. One-sample Wilcoxon rank sum tests were used to determine whether PROMIS and NIH Toolbox T scores differed from the demographically matched normative US population. Effect size calculation was performed using the effect size calculator for $t$ tests from Social Science Statistics ${ }^{17}$; all other age-dichotomized analyses were performed using GraphPad Prism, version 9.2.0. Linear and logistic regression models were performed in R, version 4.0.3 (R Foundation for Statistical Computing, Vienna, Austria). Quantitative models for continuous biomarkers were performed by linear regression. Qualitative models of patient-reported anxiety were performed by logistic regression modeling with effects expressed as ORs. Age was included as a variable in all regression models to ensure adjustment for potential confounding effects of age between continuous biomarkers or anxiety measures and the patient groups.

Study data were collected and managed using REDCap electronic data capture tools. Subjects with missing variables were eliminated from quality-of-life analyses when necessary, and no subject values were missing for biochemical markers.
For all models and statistical tests, 2 -sided $p$ values $\leq 0.05$ were considered statistically significant.

\section{Data Availability}

Anonymized data are available by request to any qualified investigator.

\section{Results}

\section{Patient Demographics and Study Groups}

We recruited a total of 64 study participants, including 56 patients experiencing neurologic symptoms attributable to COVID-19 and 8 healthy control (HC) subjects who have never been infected with SARS-CoV-2 for inclusion in this study between March 2020 and December of 2020, before the availability of COVID-19 vaccines. Study subjects were subdivided into acutely infected hospitalized patients with encephalopathy whose samples were collected concurrent to hospitalization (CE, $n=9$ ); patients who were hospitalized for pneumonia during acute infection and who have subsequently developed neuro-PASC (PNP, $\mathrm{n}=9$ ); patients who were never hospitalized, exhibiting only mild-tomoderate symptoms in acute infection, but who subsequently developed neuro-PASC (NNP, $\mathrm{n}=38)$; and HC $(\mathrm{n}=8)$ subjects who have never been infected with SARSCoV-2.

The mean age values for patients with $\mathrm{CE}$ were significantly higher than those in all other groups (74.9 \pm 15.8 years, $p<$ 0.001; Table 1), reflecting the increased severity of acute 


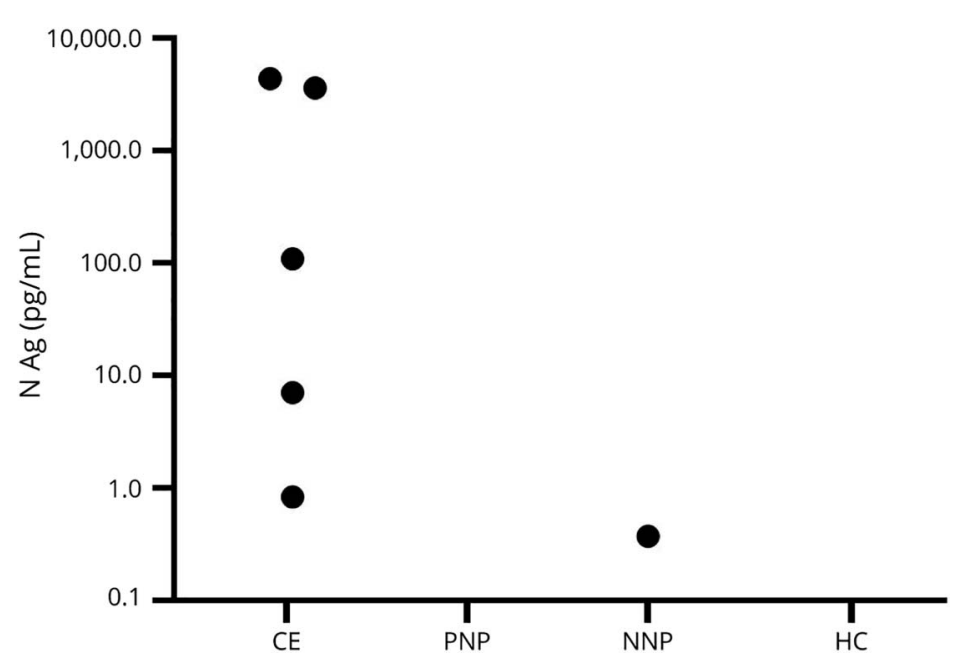

N Ag was detectable in 6 of the 64 (9.4\%) plasma samples tested ( $\mathrm{CE}=5$ of the $9[55.6 \%]$, posthospitalization neuro-PASC patients $=0 / 9$, nonhospitalized neuro-PASC patient $=1$ of the 38 [2.6\%], healthy controls $=0$ of the 8 ).
COVID-19 in older populations. Patients with CE did not have documented neurologic disease which would account for increased concentration of either $\mathrm{pNfL}$ or $\mathrm{pGFAP}$. The mean age values for the remaining study groups (PNP: $54.1 \pm 20.3$ years; NNP: $45.5 \pm 12.1$ years; HC: $38.5 \pm 10.9$ years) were not significantly different by Tukey post hoc analysis. All populations had greater female enrollment, with the exception of the PNP group. Most subjects identified as non-Hispanic White. Samples were obtained in the acute phase for patients with $\mathrm{CE}$ at a median of 20 days postsymptom onset (DPO) ( $p$ $<0.001)$, when compared with samples of patients with neuro-PASC, which were collected at 237 (PNP) and 196 $(\mathrm{NNP})$ DPO $(p=0.45)$.

\section{Biomarkers of CNS Injury in Patients With CE and Neuro-PASC}

We determined the plasma concentration of $\mathrm{pNfL}$ and $\mathrm{pGFAP}$ to assess the relationship between CNS and neurologic symptoms of SARS-CoV-2 infection and signs of neuronal injury and astrocytic activation (Table 1 and Figure 1). It is well established that $\mathrm{pNfL}$ concentration is correlated with increasing age, and therefore, we stratified our study populations into age ranges younger and older than 50 years, which was the mean age for all participants, to account for age-dependent changes. ${ }^{18}$ Acutely infected CE patients older than 50 years had significantly higher median pNfL (75.6 [60.6-269.7] pg/mL, $p=0.03)$ and pGFAP (198.9 [126.3-457.1] pg/mL, $p=0.003)$ concentrations than all other groups (Figure 1A). Neuro-PASC groups and HC groups comprising subjects younger than 50 years did not differ in the median pNfL $(p=0.83)$ or pGFAP concentration $(p=0.80)$ (Figures $1 \mathrm{~B}$ and $\mathrm{C}$ ). Neuro-PASC groups comprising patients older than 50 years did not differ in the median pNfL $(p=0.44)$ or pGFAP concentration $(p=0.97)$ (Figures $1 \mathrm{~B}$ and $\mathrm{C}$ ). Healthy control samples older than 50 years could not be included in the age-dichotomized comparisons because of low representation. We additionally performed a linear regression model for $\mathrm{pNfL}$ and $\mathrm{pGFAP}$ vs each COVID-19 patient category with adjustment for age to ensure that the observed statistical difference in pNfL and pGFAP in the CE group was not because of the higher age of this patient group. Age was not a statistically significant variable in either model of analyte levels, with a linear regression $p$ value for age of 0.79 for $\mathrm{pNfL}$ and $p=0.06$ for pGFAP. The linear regression models confirmed the agestratified findings that $\mathrm{pNfL}$ and $\mathrm{pGFAP}$ levels are expected to be significantly greater for patients with $\mathrm{CE}$ than any other group, even after adjusting for age ( $\mathrm{pNfL}$ model intercept for CE group 236.5 [95\% CI 69.4 to 403.6 ] with a multiple $\mathrm{R}^{2}$ value of 0.33 ; model intercepts for other patient groups relative to CE group: HC group -220.7 [95\% CI -348.8 to -92.6 ], NNP group -216.5 [95\% CI -316.1 to -116.9 ], and PNP group -212.9 [95\% CI -323.1 to -102.6 . pGFAP model intercept for CE group 135.4 [95\% CI -26.9 to 297.1] with a multiple $\mathrm{R}^{2}$ value of 0.38 ; model intercepts for other patient groups relative to $\mathrm{CE}$ group: HC group -147.1 [95\% CI -271.1 to -23.1$]$, NNP group -156.9 [95\% CI -253.3 to -60.5 ], and PNP group- 178.5 [95\% CI -285.2 to -71.8$])$. Additional models to include sex and body mass index (BMI) were performed. Inclusion of these variables did not affect the outcomes of our model: pNfL model intercept for CE group adjusted for age, sex, and BMI was 209.0 [95\% CI -7.03 to 425 ] with a multiple $\mathrm{R}^{2}$ value of 0.39 . Model intercepts for other groups relative to $\mathrm{CE}$ group were as follows: $\mathrm{HC}$ group -220.8 [95\% CI -349.3 to -92.3 ]; NNP group -219.5 [95\% CI -320.7 to -118.3 ]; and PNP group -226.2 [95\% CI -340.8 to -111.5 ]. Model intercept for pGFAP in CE group adjusted for age, sex, and BMI was 150.5 [95\% CI -57.8 to 358.8] with a multiple $\mathrm{R}^{2}$ value of 0.39 . Model intercepts for other groups relative to $\mathrm{CE}$ were as follows: $\mathrm{HC}$ group -152.2 [95\% CI -276.1 to -28.3 ]; NNP group -161 [95\% CI -259.0 to $-63.8]$; and PNP group -190.8 [95\% CI -301.3 to -80.3$]$.

To determine the contribution of glial activation when compared with neuronal damage, we calculated a neuroglial score 
Table 2 Symptoms Attributed to COVID-19

\begin{tabular}{|c|c|c|c|}
\hline & PNP $\mathbf{n}=9$ & NNP $n=38$ & $p$ Value \\
\hline Percentage recovered since baseline (median [IQR]) & $75[60-90]$ & $60[50-70]$ & 0.05 \\
\hline Number of neurologic symptoms attributed to PASC (median [IQR]) & $3[1.5-4]$ & $5.5[4-7]$ & 0.002 \\
\hline \multicolumn{4}{|l|}{ Neurologic symptoms, n (\%) } \\
\hline Brain fog & $8(88.9)$ & $35(92.1)$ & 0.76 \\
\hline Headache & $4(44.4)$ & $28(73.7)$ & 0.09 \\
\hline Myalgia & $3(33.3)$ & $26(68.4)$ & 0.05 \\
\hline Dysgeusia & $2(22.2)$ & 27 (71.7) & 0.006 \\
\hline Numbness/tingling & $3(33.3)$ & $17(44.7)$ & 0.53 \\
\hline Anosmia & $3(33.3)$ & $25(65.8)$ & 0.07 \\
\hline Dizziness & $2(22.2)$ & $19(50.0)$ & 0.13 \\
\hline Tinnitus & $2(22.2)$ & $14(36.8)$ & 0.41 \\
\hline Pain in areas other than chest & $0(0.0)$ & $17(44.7)$ & $<0.001$ \\
\hline Blurred vision & $2(22.2)$ & $11(28.9)$ & 0.69 \\
\hline \multicolumn{4}{|l|}{ Other symptoms, n (\%) } \\
\hline Fatigue & $5(55.6)$ & $36(94.7)$ & 0.03 \\
\hline Shortness of breath & $7(77.7)$ & $21(55.3)$ & 0.22 \\
\hline Depression/anxiety & $6(66.6)$ & $26(68.4)$ & 0.92 \\
\hline Insomnia & $3(33.3)$ & $21(55.3)$ & 0.24 \\
\hline Chest pain & $1(11.1)$ & $14^{\mathrm{a}}(37.8)$ & 0.13 \\
\hline Variations in heart rate and blood pressure & $2(22.2)$ & $12(31.6)$ & 0.58 \\
\hline GI symptoms & $2(22.2)$ & $14(36.8)$ & 0.41 \\
\hline
\end{tabular}

Abbreviations: NNP $=$ nonhospitalized neuro-PASC patients; PNP = posthospitalization neuro-PASC patients .

Bold values indicate any $p$ values $<0.05$ and were considered significant.

${ }_{\mathrm{a}}=37$.

based on the ratio of pGFAP to $\mathrm{pNfL}$, as previously described for other neurologic conditions. ${ }^{5,19,20}$ Higher scores indicate that astroglial activation is dominant and lower scores indicate that neuronal injury is dominant. All age-stratified groups had similar neuroglial scores except for a trend toward lower scores in CE patients older than 50 years $(p=0.07)$, suggesting that there may be greater neuronal damage relative to astrocytic activation in acutely encephalopathic when compared with patients with neuro-PASC. A linear regression model of the neuroglial score confirmed that age was not statistically associated with neuroglial score $(p=0.24)$, and that although neuroglial scores tended to be lower for patients with $\mathrm{CE}$, they did not differ significantly between groups.

\section{SARS-CoV-2 Nucleocapsid Antigen Is Present in the Blood of Acutely Infected Patients With} Encephalopathy and a Patient With Neuro-PASC To determine whether there was correlation between markers of CNS injury and expression of viral antigen, we tested the same samples for the presence of SARS-CoV-2 nucleocapsid $(\mathrm{N} \mathrm{Ag})$ protein. We found that 5 of the 9 patients with $\mathrm{CE}$ (55.6\%) had detectable titers of N Ag in their blood (Figure 2). There was no correlation with pNfL, pGFAP, neuroglial score, or sample DPO and N Ag titer (data not shown). Of interest, we identified 1 individual from the NNP group (1/38, 2.6\%) who had detectable $\mathrm{N} \mathrm{Ag}(0.37 \mathrm{pg} / \mathrm{mL})$ in the plasma from a blood sample obtained 3 weeks after symptom onset in a patient who maintains neuro-PASC symptoms 350 days postinfection to date, suggesting that there is a productive reservoir of antigenemia or infection in this individual 3 weeks postsymptoms onset. No PNP or HC individuals had detectable plasma $\mathrm{N} \mathrm{Ag}$.

\section{Symptoms Associated With COVID-19, Quality-of-} Life Measures, and Standardized Cognitive Tests

We next sought to determine whether there was an association between pNfL, pGFAP, and neuroglial scores with individual symptoms, changes in quality of life, or cognitive performance associated with neuro-PASC. 


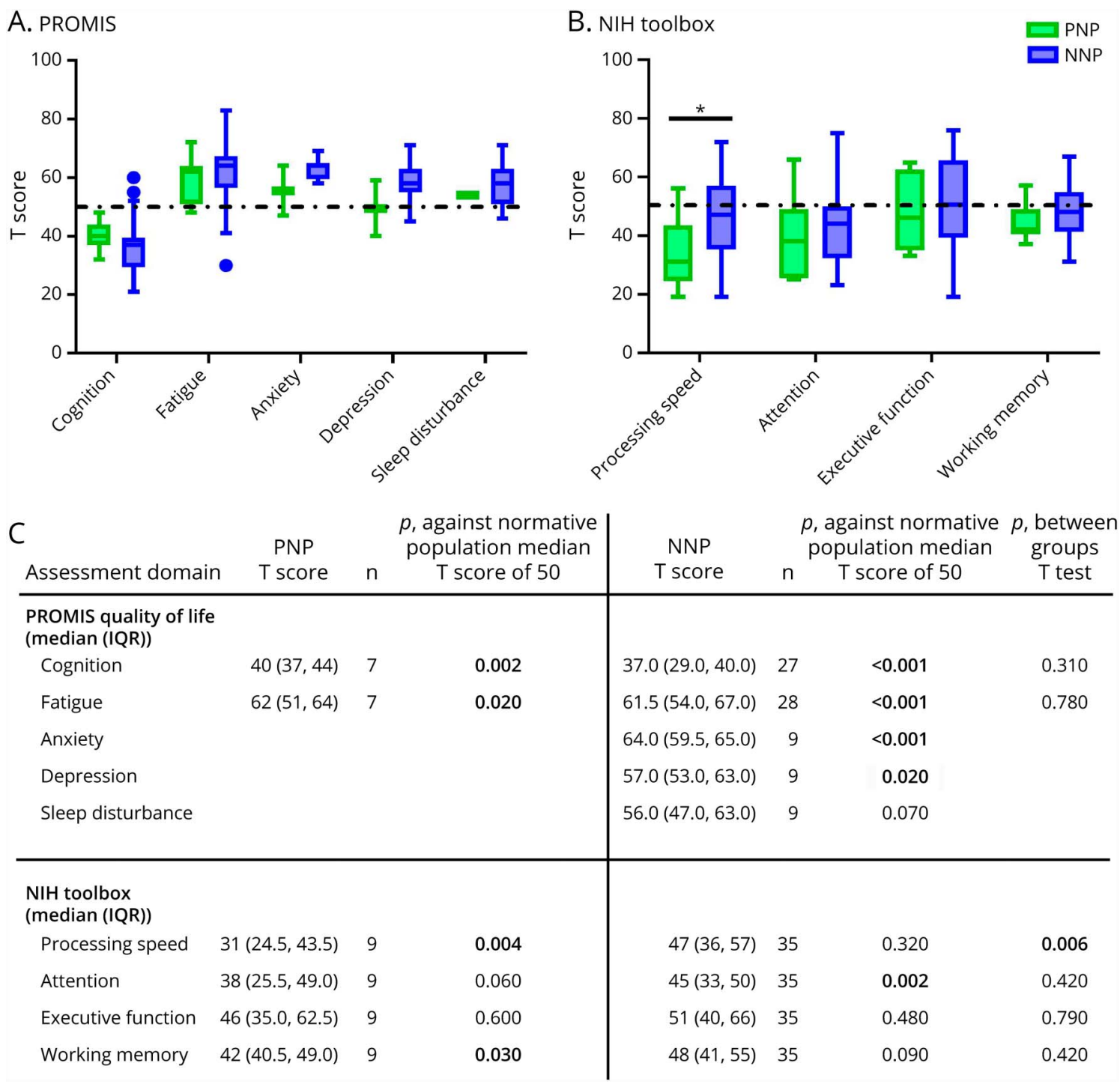

Normalized and demographic-matched T score values for Patient-Reported Outcomes Measurement Information System (PROMIS-57; A) quality of life and $\mathrm{NIH}$ Toolbox (B) cognitive assessment for posthospitalized neuro-PASC patients (PNP) and nonhopitalized neuro-PASC patients (NNP). United States normative population T score mean/median of $50(\delta=10)$ is indicated by a broken black line. Scores lower than 50 for cognition, processing speed, attention, executive function, and working memory indicate poor outcomes. Scores higher than 50 for fatigue, anxiety, depression, and sleep disturbance indicate poor outcomes. One-sample 2 -tailed $t$ test $p$ values between patient group and normative population and between-group 2 -tailed $t$ test are provided in the table. * $p \leq 0.05$.

Neurologic evaluation was performed for all subjects enrolled in the PNP and NNP groups in the Northwestern University hospital Neuro-COVID-19 clinic. Symptom frequency is described in Table 2. On average, PNP patients had a median of 3 neurologic symptoms, whereas those who had a mild initial presentation, which did not require hospitalization, reported a higher number of neurologic symptoms (NNP $M=5.5 ; p=0.002$ ). The most frequently reported neurologic symptoms among both groups were brain fog, headaches, and myalgias. Most neurologic symptoms were reported with similar frequency between neuro-PASC groups with the exception of dysgeusia and pain in areas other than the chest, both of which were reported infrequently in PNP patients $(p=0.006$ and $p<0.001$, respectively). Non-neurologic symptoms most frequently reported in all groups were fatigue, shortness of breath, and depression/anxiety. Of interest, NNP patients reported fatigue more frequently than PNP patients $(p=$ 0.03 ), whereas all other non-neurologic symptoms were reported with similar frequency between groups.

Neuro-PASC impacts on quality of life and cognitive performance were analyzed using PROMIS and NIH Toolbox $\mathrm{T}$ scores, respectively (Figures 3, A and B). Within-group performance was compared with national standardized values for each test. Both groups showed significantly worse patientreported cognition and fatigue scores than the demographically matched cohorts (Figure 3C). NNP patients had significantly worse patient-reported anxiety scores than the national averages and significantly worse rates of patient- 

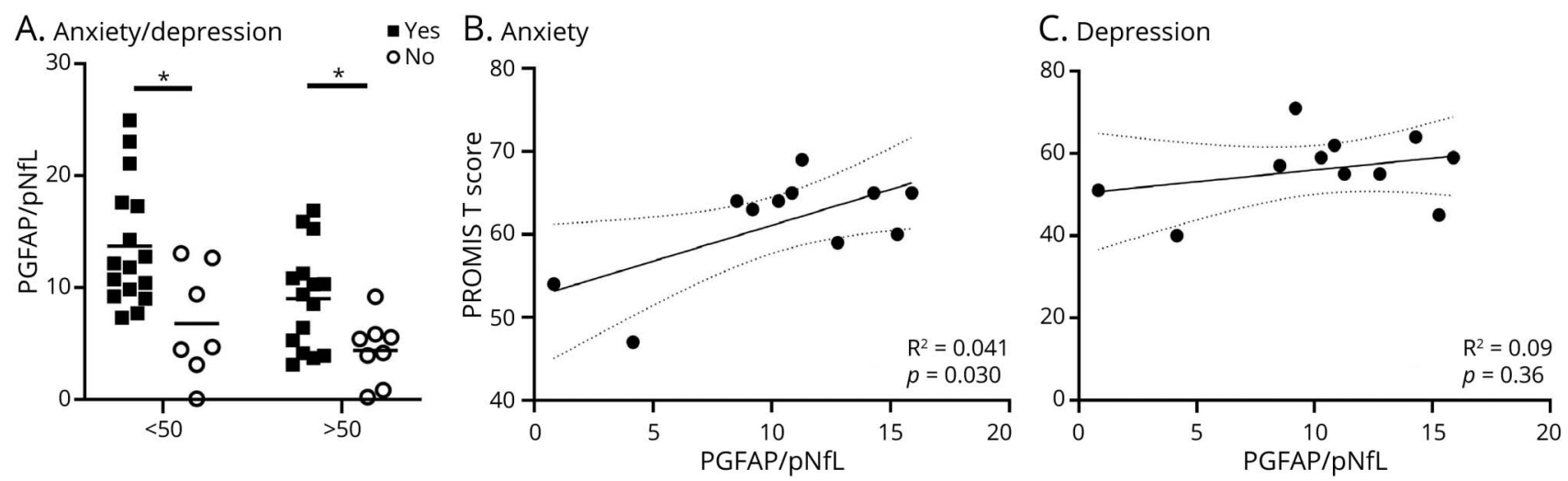

Neuro-PASC patients (posthospitalization neuro-PASC patients and nonhospitalized neuro-PASC patients combined) presenting with anxiety and/or depression (A) in the Neuro-Covid-19 clinic showed higher neuroglial scores when compared with neuro-PASC patients who exhibited neither anxiety nor depression in both younger than 50-year age-group and older than50-year age-group (younger than 50 years: yes, $n=17 ; n o, n=7, p=0.01$, Hedges' $g$ effect size $=1.37$; older than 50 years: yes, $\mathrm{n}=15 ; \mathrm{no} \mathrm{n}=8, p=0.02$, Hedges' $\mathrm{g}$ effect size $=1.17$; two-tailed T tests). Quantitative PROMIS-57 T scores for anxiety (B) but not depression (C) showed linear correlation with increasing glial activation predominance at the time of sample (anxiety $\mathrm{T}$ score: $\mathrm{n}=11, \mathrm{R} 2=0.41, p=0.03$; depression T score: $\mathrm{n}=11, \mathrm{R} 2=0.09, p=0.36$; two-tailed Pearson correlation). ${ }^{*} p \leq 0.05$.

reported depression. PNP measures were not compared with national averages for anxiety, depression, or sleep disturbance because of low enrollment for these measures $(n=2)$. No difference was observed in between-group patient-reported values for any domain tested.

NIH Toolbox measures showed that PNP patients, but not NNP patients, performed significantly worse in processing speed and working memory than the national averages. Conversely, NNP patients performed worse in attention than the national average, whereas PNP patients only showed a trend toward significance (NNP, $p=0.002$; PNP, $p=0.06$ ). Overall, only processing speed was different between groups, with PNP patients showing significantly worse measures than NNP patients $(p=0.006)$.

Collectively, these data suggest that patients with neuro-PASC have largely similar decreased quality of life associated with neuro-long-hauler symptoms regardless of severity of the acute phase of their disease, and both PNP and NNP patients exhibit cognitive dysfunction, albeit affecting different domains.

\section{Neuroglial Score Shows Associations Between Astrocytic Predominance and Anxiety in Neuro- PASC Patients}

All the quantitative (Table 2) and qualitative (Figure 3) variables were compared with pNfL, pGFAP, and neuroglial scores to determine whether either biomarker or the involvement of axonal damage or astrocytic activation predominance associated with outcomes within groups for both age-stratified subsets (data not shown).

We observed an association between patient-reported anxiety and/or depression at the time of the outpatient clinic visit and lower neuroglial score in both young and older patients with neuro-PASC (PNP and NNP patients combined)
(Figure 4A). Age in anxiety-stratified or depression-stratified groups was statistically similar: the mean age for patients younger than 50 years who experienced anxiety/depression was 36.5 years, whereas those with no anxiety/depression had a mean age of 34 years $(p=0.56)$; the mean age for those older than 50 years with anxiety/depression was 59.2 years, whereas those with no anxiety/depression had a mean age of 57.9 years $(p=0.69)$. Logistic regression accounting for age confirmed the association between increasing neuroglial score and greater qualitative experience of anxiety/depression, with an odds ratio of 1.32 (95\% CI 1.12, 1.64, $p=0.004)$ even with adjustment for age.

To determine the respective contributions of anxiety and depression with higher neuroglial scores, we analyzed patientreported quantitative values obtained by PROMIS testing within 1 week of sample date, which showed a significant positive correlation between neuroglial score and anxiety $\mathrm{T}$ score $(\mathrm{Rho}=0.64$, $p=0.03$; age-adjusted linear regression $\beta=0.87$ PROMIS anxiety increase per neuroglial unit increase, $p=0.04$ with a multiple $\mathrm{R}^{2}$ of $0.41)$ but not PROMIS depression T score ( $\mathrm{Rho}=0.30, p=0.36$; age-adjusted linear regression $\beta=0.58$ PROMIS depression increase per neuroglial unit increase, $p=0.38$, with a multiple $\mathrm{R}^{2}$ of 0.13 ), suggesting that anxiety is associated with increasing neuroglial score in patients with neuro-PASC (Figures 4, B and C). pGFAP concentration independently did not reach significance with qualitative determination of anxiety/depression but did correlate with PROMIS anxiety $\mathrm{T}$ score ( $\mathrm{Rho}=0.62$, multiple $\mathrm{R}^{2}=0.38, p=0.04$; data not shown). Collectively, these data suggest that astrocytic activation is associated with symptoms of anxiety in patients with neuro-PASC.

\section{Discussion}

Our results confirm previously published findings that patients hospitalized with severe COVID-19 have elevated biomarkers 
of CNS injury in their blood. ${ }^{6,21-25}$ This finding therefore supports the body of literature showing that COVID-19 neurologic sequelae in the acute phase are accompanied by damage to the CNS despite the fact that direct SARS-CoV-2 infection of the brain parenchyma remains a matter of debate. ${ }^{26}$ In severely ill patients, the possible causes of CNS injury include hypoxemia, vasculopathy, coagulopathy, or systemic inflammation leading to blood-brain barrier disruption. However, elevation of serum (s)GFAP has also been observed in acute moderate cases and elevation of serum (s) NfL in acute mild-tomoderate cases of COVID-19. ${ }^{6,23,24}$ Collectively, these findings suggest that CNS damage may occur in patients with COVID19 presenting with a wide range of acute disease severity.

The conceptual basis for analyzing our patient cohorts using a neuroglial score is derived from the use of these markers in differentiating between relapsing-remitting and primary progressive multiple sclerosis (MS) and between neuromyelitis optica spectrum disorder (NMOSD) and MS. In these cases, it has been shown that predominance of glial activation correlates with progressive forms of MS, with NMOSD when compared with MS, with $\alpha$-aquaporin-4 antibody predominance in NMOSD, and with increasing disability associated with MS and NMOSD, collectively suggesting that the neuroglial score used in this study is associated with distinct underlying pathophysiology in those disease processes. ${ }^{4,5,19,20}$

Within-group comparisons of patients with neuro-PASC revealed that there was a significant difference in neuroglial scores for those experiencing depression and/or anxiety as a symptom of neuro-PASC. Additional quantitative measures of anxiety and depression revealed that there was a linear relationship between increasing neuroglial scores and anxiety, but not depression, in patients with neuro-PASC.

Astrocytic involvement in neuropsychiatric disorders is a developing field of study. Functional differences have been shown in astrocytes from animal models of depression, and similar changes have been observed in postmortem tissues obtained from suicide victims. ${ }^{27}$ Among other changes, astrocytic glutamate transporter 1 (GLT-1/EAAT2) expression decreases in animal models of stress and anxiety, and its expression is increased by exposure to antidepressants. ${ }^{28,29}$ Furthermore, transgenic mice that had conditional expression of astrocytic GLT-1 showed greater resilience to anxiety and depression. ${ }^{30}$ These and other data show that astrocytes play a role in anxiety and corroborate our neuroglial score results, which suggest that astrocytic activation predominates in neuro-PASC patients experiencing anxiety.

Previous coronaviruses have been implicated in neuropsychiatric disorders such as depression and anxiety in a variety of ways. Seropositivity for common cold alphacoronavirus strain HCoV-NL63 was shown to be elevated in new-onset psychosis and more frequent in individuals with mood disorders such as major depressive disorder. ${ }^{31,32}$ Furthermore, survivors of the SARS-CoV-1 epidemic in 2003 showed an increase in prevalence of psychiatric disorders from $3.3 \%$ before infection to $42.5 \%$ at 40 months postinfection, with the most common diagnoses of post-traumatic stress disorder (54.5\%) and depression $(39.0 \%) .^{33}$ Large-scale surveillance for neuropsychiatric conditions after COVID-19 should continue, and our data suggest that biochemical markers of CNS damage may help to identify the mechanistic cause of neuropsychiatric symptoms in neuro-PASC patients. Nucleocapsid antigenemia suggest prolonged SARS-CoV-2 infection in some nonhospitalized neuro-PASC patients.

The underlying mechanisms of PASC remain unclear. We and others have shown that most of the non-hospitalized patients with neuro-PASC are women, who are more likely than men to develop autoimmune conditions. Furthermore, patients with neuro-PASC have higher frequency of pre-existing autoimmune conditions before COVID-19, suggesting that PASC may be a postinfectious, autoimmune phenomenon. ${ }^{10}$ However, prolonged viral shedding has been documented in some patients after the acute phase of COVID-19, and the T-cell response to SARS-CoV-2 in non-hospitalized neuro-PASC patients is consistent with that seen in the setting of persistent antigenic stimulation. ${ }^{34}$ Our data indicate that nucleocapsid antigenemia may occur at least 3 weeks after symptom onset in a nonhospitalized patient who developed neuro-PASC. Viral RNA in fecal samples has been detected for greater than 5 weeks in hospitalized patients who no longer had detectable viral RNA in nasopharyngeal or throat swab samples, suggesting that the gut may function as a reservoir of viral replication with longer duration than the respiratory tract. ${ }^{35}$ The GI tract contains a large collection of specialized immune cells. Prolonged viral replication in this compartment might provoke inflammatory cascades ultimately inducing chronic inflammation or the induction of autoimmunity through epitope spreading or bystander activation. Gut-associated SARS-CoV-2 replication and antigen persistence has yet to be studied in nonhospitalized patients or in patients with neuro-PASC, but should be pursued. Future work should focus on larger surveillance for the presence of Nucleocapsid Ag in the periphery, including through repeated fecal and nasopharyngeal sampling, assessing for nucleocapsidspecific T-cell activation, and should determine whether an ongoing productive infection with SARS-CoV-2 is occurring in hidden reservoirs such as the gut. ${ }^{36}$

Our study has limitations, primarily its limited sample size. The pilot data collected for this study included small HC and $\mathrm{CE}$ groups. Continuing investigations should emphasize larger sample size and age matching across all groups to increase the statistical power of the observation. We were unable to include patients without post-COVID neurologic symptoms because of underrepresentation in our NeuroCOVID-19 clinic. Future work should include this patient population to determine whether SARS-CoV-2 infection affects the levels of plasma biomarkers in the absence of neurologic sequelae. Patients had a single measurement of $\mathrm{pNfL}$, pGFAP, and plasma SARS-CoV-2 Nucleocapsid antigen, and patients with neuro-PASC were tested at a median of approximately 7 months postsymptoms onset, when they felt 
already approximately $70 \%$ recovered compared with their pre-COVID-19 baseline by their own report. It is therefore possible that higher plasma concentrations of CNS biomarkers and more frequent detection of SARS-CoV-2 N Ag could be found in earlier stages of neuro-PASC. PROMIS questions for anxiety, depression, and sleep disturbance were added to our standard panel in January 2021 and therefore were not available for all participants. A prospective study, including a larger number of patients including those with no neuro-PASC symptoms, is needed to determine the dynamic evolution of CNS biomarkers and N Ag shedding in blood or other bodily fluids over time.

It has been estimated that approximately one-third of all patients with COVID-19 develop PASC of variable duration, and neurologic symptoms are among the most debilitating manifestations of PASC. ${ }^{9}$ With more than 48 million documented infections since the beginning of the pandemic in the United States, it is likely that more than 15 million individuals have been or are currently affected by neuro-PASC in the United States alone and tens of millions in the world. ${ }^{37,38}$ This would make neuro-PASC the third most prevalent neurologic disorder in the United States behind only tension and migraine headaches and ahead of stroke, Alzheimer disease and other dementias, spinal cord and traumatic brain injuries, and idiopathic epilepsy. ${ }^{39}$ Longitudinal studies are needed to evaluate persistent SARS-CoV-2 infection and CNS damage in patients with neuro-PASC over time and the long-term cognitive impact of COVID-19 in this large patient population.

\section{Study Funding}

The authors report no targeted funding.

\section{Disclosure}

The authors declare no competing interests. Go to Neurology.org/NN for full disclosures.

\section{Publication History}

Received by Neurology: Neuroimmunology \& Neuroinflammation October 4, 2021. Accepted in final form January 24, 2022. Submitted and externally peer reviewed. The handling editor was Raquel SánchezValle, MD, PhD.

\section{Appendix Authors}

\begin{tabular}{|c|c|c|}
\hline Name & Location & Contribution \\
\hline $\begin{array}{l}\text { Barbara A. } \\
\text { Hanson, BA }\end{array}$ & $\begin{array}{l}\text { Ken and Ruth Davee } \\
\text { Department of Neurology, } \\
\text { Feinberg School of } \\
\text { Medicine, Northwestern } \\
\text { University; Rush Medical } \\
\text { College, Chicago, IL }\end{array}$ & $\begin{array}{l}\text { Drafting/revision of the } \\
\text { article for content, including } \\
\text { medical writing for content; } \\
\text { major role in the acquisition } \\
\text { of data; study concept or } \\
\text { design; and analysis or } \\
\text { interpretation of data }\end{array}$ \\
\hline $\begin{array}{l}\text { Lavanya } \\
\text { Visvabharathy, } \\
\text { PhD }\end{array}$ & $\begin{array}{l}\text { Ken and Ruth Davee } \\
\text { Department of Neurology, } \\
\text { Feinberg School of } \\
\text { Medicine, Northwestern } \\
\text { University, Chicago, IL }\end{array}$ & $\begin{array}{l}\text { Drafting/revision of the } \\
\text { article for content, including } \\
\text { medical writing for content; } \\
\text { major role in the acquisition } \\
\text { of data; study concept or } \\
\text { design; and analysis or } \\
\text { interpretation of data }\end{array}$ \\
\hline
\end{tabular}

Appendix (continued)

\begin{tabular}{lll}
\hline Name & Location & Contribution \\
\hline Sareen T. Ali, BA & $\begin{array}{l}\text { Ken and Ruth Davee } \\
\text { Department of Neurology, } \\
\text { Feinberg School of } \\
\text { Medicine, Northwestern } \\
\text { University, Chicago, IL }\end{array}$ & $\begin{array}{l}\text { Drafting/revision of the } \\
\text { article for content, including } \\
\text { medical writing for content, } \\
\text { and major role in the } \\
\text { acquisition of data }\end{array}$ \\
\hline $\begin{array}{l}\text { Anthony K. } \\
\text { Kang, BS }\end{array}$ & $\begin{array}{l}\text { Ken and Ruth Davee } \\
\text { Department of Neurology, } \\
\text { Feinberg School of } \\
\text { Medicine, Northwestern } \\
\text { University, Chicago, IL }\end{array}$ & $\begin{array}{l}\text { Drafting/revision of the } \\
\text { article for content, including } \\
\text { medical writing for content, } \\
\text { andajor role in the } \\
\text { acquisition of data }\end{array}$ \\
\hline
\end{tabular}

Tulsi R. Patel, Ken and Ruth Davee Drafting/revision of the

BA Department of Neurology, article for content, including Feinberg School of medical writing for content, Medicine, Northwestern and major role in the University, Chicago, IL acquisition of data

\begin{tabular}{lll}
\hline Jeffrey R. Clark, & Ken and Ruth Davee & Drafting/revision of the \\
BA & Department of Neurology, & article for content, including \\
& Feinberg School of & medical writing for content; \\
& Medicine, Northwestern & $\begin{array}{l}\text { major role in the acquisition } \\
\text { of data }\end{array}$ \\
& University, Chicago, IL & .
\end{tabular}

Patrick H. Lim, Ken and Ruth Davee Drafting/revision of the

MS Department of Neurology, article for content, including Feinberg School of medical writing for content, Medicine, Northwestern and major role in the University, Chicago, IL acquisition of data

\begin{tabular}{lll}
\hline $\begin{array}{l}\text { Zachary S. } \\
\text { Orban, BS }\end{array}$ & $\begin{array}{l}\text { Ken and Ruth Davee } \\
\text { Department of Neurology, } \\
\begin{array}{l}\text { Feinberg School of } \\
\text { Medicine, Northwestern } \\
\text { University, Chicago, IL }\end{array}\end{array}$ & $\begin{array}{l}\text { Drafting/revision of the } \\
\text { article for content, including } \\
\text { medical writing for content, } \\
\text { and major role in the } \\
\text { acquisition of data }\end{array}$ \\
\hline $\begin{array}{l}\text { Soyoon S. } \\
\text { Hwang, PhD }\end{array}$ & $\begin{array}{l}\text { Quanterix Corporation, } \\
\text { Billerica, MA }\end{array}$ & $\begin{array}{l}\text { Drafting/revision of the } \\
\text { article for content, including } \\
\text { medical writing for content, } \\
\text { and major role in the } \\
\text { acquisition of data }\end{array}$ \\
\end{tabular}

Dawn Mattoon, Quanterix Corporation, Drafting/revision of the PhD $\quad$ Billerica, MA article for content, including medical writing for content, andajor role in the acquisition of data

\begin{tabular}{lll}
\hline $\begin{array}{l}\text { Ayush Batra, } \\
\text { MD }\end{array}$ & $\begin{array}{l}\text { Ken and Ruth Davee } \\
\text { Department of Neurology, } \\
\begin{array}{l}\text { Feinberg School of } \\
\text { Medicine, Northwestern } \\
\text { University, Chicago, IL }\end{array}\end{array}$ & $\begin{array}{l}\text { Drafting/revision of the } \\
\text { article for content, including } \\
\text { medical writing for content, } \\
\text { and analysis or } \\
\text { interpretation of data }\end{array}$ \\
$\begin{array}{lll}\text { Eric M. Liotta, } \\
\text { MD, MS }\end{array}$ & $\begin{array}{l}\text { Ken and Ruth Davee } \\
\text { Department of Neurology, } \\
\text { Feinberg School of } \\
\text { Medicine, Northwestern } \\
\text { University, Chicago, IL }\end{array}$ & $\begin{array}{l}\text { Drafting/revision of the } \\
\text { article for content, including } \\
\text { medical writing for content, } \\
\text { and analysis or } \\
\text { interpretation of data }\end{array}$ \\
\hline $\begin{array}{l}\text { Igor J. Koralnik, } \\
\text { MD }\end{array}$ & $\begin{array}{l}\text { Ken and Ruth Davee } \\
\text { Department of Neurology, } \\
\text { Feinberg School of } \\
\text { Medicine, Northwestern } \\
\text { University, Chicago, IL }\end{array}$ & $\begin{array}{l}\text { Drafting/revision of the } \\
\text { including medical writing for } \\
\text { content; major role in the } \\
\text { acquisition of data; study } \\
\text { concept or design; and } \\
\text { analysis or interpretation of } \\
\text { data }\end{array}$ \\
& &
\end{tabular}

\section{References}

1. COVID-19 Dashboard by the Center for Systems Science and Engineering (CSSE) at Johns Hopkins University \& Medicine. Johns Hopkins University \& Medicine. Accessed November 26, 2021. coronavirus.jhu.edu/map.html.

2. Koralnik IJ, Tyler KL. COVID-19: a global threat to the nervous system. Ann Neurol. 2020/07;88(1):1-11. 
3. Liotta EM, Batra A, Clark JR, et al Frequent neurologic manifestations and encephalopathy-associated morbidity in Covid-19 patients. Ann Clin Translational Neurol. 2020;7(11):2221-2230.

4. Abdelhak A, Huss A, Kassubek J, Tumani H, Otto M. Serum GFAP as a biomarker for disease severity in multiple sclerosis. Sci Rep. 2018;8(1):14798.

5. Watanabe M, Nakamura Y, Michalak Z, et al. Serum GFAP and neurofilament light as biomarkers of disease activity and disability in NMOSD. Neurology. 2019;93(13):e1299-e1311.

6. Ameres M, Brandstetter S, Toncheva AA, et al Association of neuronal injury blood marker neurofilament light chain with mild-to-moderate COVID-19. J Neurol 2020; 267(12):3476-3478. doi: doi:10.1007/s00415-020-10050-y

7. Cooper J, Stukas S, Hoiland RL, et al Quantification of neurological blood-based biomarkers in critically ill patients with coronavirus disease 2019. Crit Care Explor. 2020;2(10):e0238.

8. NIH announces research opportunities to study. Long COVID. National Institutes of Health Accessed November 26, 2021. ninr.nih.gov/newsandinformation/newsandnotes/pasc-initiative.

9. Taquet M, Dercon Q, Luciano S, Geddes JR, Husain M, Harrison PJ. Incidence, cooccurrence, and evolution of long-COVID features: a 6-month retrospective cohort study of 273,618 survivors of COVID-19. PLoS Med. 2021;18(9):e1003773.

10. Graham EL, Clark JR, Orban ZS, et al Persistent neurologic symptoms and cognitive dysfunction in non-hospitalized Covid-19 "long haulers". Ann Clin Transl Neurol. 2021;8(5):1073-1085

11. Lai JS, Cella D, Choi S, et al How item banks and their application can influence measurement practice in rehabilitation medicine: a PROMIS fatigue item bank example. Arch Phys Med Rehabil. 2011;92(10 Suppl):S20-S27.

12. Lai J-S, Wagner LI, Jacobsen PB, Cella D. Self-reported cognitive concerns and abilities: two sides of one coin? Psychooncology. 2014;23(10):1133-1141.

13. Gershon RC, Wagster MV, Hendrie HC, Fox NA, Cook KF, Nowinski CJ. NIH toolbox for assessment of neurological and behavioral function. Neurology. 2013; 80(11 Suppl 3):S2-S6.

14. Heaton RK, Akshoomoff N, Tulsky D, et al Reliability and validity of composite scores from the NIH Toolbox Cognition Battery in adults. J Int Neuropsychol Soc. 2014; 20(6):588-598.

15. Weintraub S, Dikmen SS, Heaton RK, et al Cognition assessment using the NIH Toolbox. Neurology. 2013;80(11 Suppl 3):S54--64.

16. Weintraub S, Dikmen SS, Heaton RK, et al The cognition battery of the NIH toolbox for assessment of neurological and behavioral function: validation in an adult sample. J Int Neuropsychol Soc. 2014;20(6):567-578.

17. Social Science Statistics. socscistatistics.com.

18. Valentino P, Marnetto F, Martire S, et al. Serum neurofilament light chain levels in healthy individuals: a proposal of cut-off values for use in multiple sclerosis clinical practice. (2211-0356 (Electronic)).

19. Chang X, Huang W, Wang L, et al Serum neurofilament light and GFAP are associated with disease severity in inflammatory disorders with aquaporin-4 or myelin oligodendrocyte glycoprotein antibodies. Front Immunol. 2021;12:647618.

20. Huss A, Otto M, Senel M, Ludolph AC, Abdelhak A, Tumani H. A score based on NfL and glial markers may differentiate between relapsing-remitting and progressive MS course. Front Neurol. 2020;11:608.

21. Chung HY, Neu C, Wickel J, Kuckertz SL, Coldewey SM. Neurofilament light chain in patients with COVID-19 and bacterial pneumonia. Ann Neurol. 2021;90(1):174-175.
22. Geis T, Brandstetter S, Toncheva AA, et al. Serum neurofilament light chain (sNfL) values in a large cross-sectional population of children with asymptomatic to moderate COVID-19. J Neurol. 2021;268(11):3969-3974.

23. Kanberg N, Ashton NJ, Andersson LM, et al Neurochemical evidence of astrocytic and neuronal injury commonly found in COVID-19. Neurology. 2020;95(12): e1754-e1759.

24. Kanberg N, Simren J, Eden A, et al. Neurochemical signs of astrocytic and neuronal injury in acute COVID-19 normalizes during long-term follow-up. EBioMedicine. 2021;70:103512.

25. Sutter R, Hert L, De Marchis GM, et al Serum neurofilament light chain levels in the intensive care unit: comparison between severely ill patients with and without coronavirus disease 2019. Ann Neurol. 2021;89(3):610-616

26. Jakhmola S, Indari O, Chatterjee S, Jha HC. SARS-CoV-2, an underestimated pathogen of the nervous system. SN Compr Clin Med. 2020;2(11):2137-2146.

27. Kim R, Healey KL, Sepulveda-Orengo MT, Reissner KJ. Astroglial correlates of neuropsychiatric disease: from astrocytopathy to astrogliosis. Prog Neuropsychopharmacol Biol Psychiatry. 2018;87(Pt A):126-146.

28. Zink M, Vollmayr B, Gebicke-Haerter PJ, Henn FA. Reduced expression of glutamate transporters vGluT1, EAAT2 and EAAT4 in learned helpless rats, an animal model of depression. Neuropharmacology. 2010;58(2):465-473.

29. Chen JX, Yao LH, Xu BB, et al. Glutamate transporter 1-mediated antidepressant-like effect in a rat model of chronic unpredictable stress. J Huazhong Univ Sci Technolog Med Sci. 2014;34(6):838-844.

30. Jia YF, Wininger K, Ho AM, Peyton L, Baker M, Choi DS. Astrocytic glutamate transporter 1 (GLT1) deficiency reduces anxiety- and depression-like behaviors in mice. Front Behav Neurosci. 2020;14:57

31. Okusaga $\mathrm{O}$, Yolken $\mathrm{RH}$, Langenberg $\mathrm{P}$, et al. Association of seropositivity for influenza and coronaviruses with history of mood disorders and suicide attempts. J Affect Disord. 2011;130(1-2):220-225.

32. Severance EG, Dickerson FB, Viscidi RP, et al. Coronavirus immunoreactivity in individuals with a recent onset of psychotic symptoms. Schizophr Bull. 2011;37(1): 101-107

33. Lam MH, Wing YK, Yu MW, et al Mental morbidities and chronic fatigue in severe acute respiratory syndrome survivors: long-term follow-up. Arch Intern Med. 2009; 169(22):2142-2147.

34. Visvabharathy L, Hanson B, Orban Z, et al. Neuro-COVID long-haulers exhibit broad dysfunction in $\mathrm{T}$ cell memory generation and responses to vaccination. medRxiv. 2021. doi: $10.1101 / 2021.08 .08 .21261763$

35. Wu Y, Guo C, Tang L, et al. Prolonged presence of SARS-CoV-2 viral RNA in faecal samples. Lancet Gastroenterol Hepatol. 2020;5(5):434-435.

36. Gupta S, Parker J, Smits S, Underwood J, Dolwani S. Persistent viral shedding of SARS-CoV-2 in faeces - a rapid review. Colorectal Dis. 2020;22(6):611-620.

37. Baig AM. Chronic COVID syndrome: need for an appropriate medical terminology for long- COVID and COVID long-haulers. J Med Virol. 2021;93(5):2555-2556.

38. Nalbandian A, Sehgal K, Gupta A, et al. Post-acute COVID-19 syndrome. Nat Med. 2021;27(4):601-615.

39. Collaborators GUND, Feigin VL, Vos T, et al. Burden of neurological disorders across the US from 1990-2017: a global burden of disease study. JAMA Neurol. 2021;78(2): 165-176. 


\title{
Neurology \\ Neuroimmunology \& Neuroinflammation
}

\author{
Plasma Biomarkers of Neuropathogenesis in Hospitalized Patients With COVID-19 \\ and Those With Postacute Sequelae of SARS-CoV-2 Infection \\ Barbara A. Hanson, Lavanya Visvabharathy, Sareen T. Ali, et al. \\ Neurol Neuroimmunol Neuroinflamm 2022;9; \\ DOI 10.1212/NXI.0000000000001151
}

This information is current as of March 7, 2022

Updated Information \&

Services

References

Citations

Subspecialty Collections

Permissions \& Licensing

Reprints including high resolution figures, can be found at:

http://nn.neurology.org/content/9/3/e1151.full.html

This article cites 35 articles, 1 of which you can access for free at: http://nn.neurology.org/content/9/3/e1151.full.html\#\#ref-list-1

This article has been cited by 4 HighWire-hosted articles:

http://nn.neurology.org/content/9/3/e1151.full.html\#\#otherarticles

This article, along with others on similar topics, appears in the following collection(s):

COVID-19

http://nn.neurology.org//cgi/collection/covid_19

Information about reproducing this article in parts (figures,tables) or in its entirety can be found online at:

http://nn.neurology.org/misc/about.xhtml\#permissions

Information about ordering reprints can be found online:

http://nn.neurology.org/misc/addir.xhtml\#reprintsus

Neurol Neuroimmunol Neuroinflamm is an official journal of the American Academy of Neurology.

Published since April 2014, it is an open-access, online-only, continuous publication journal. Copyright

Copyright $\odot 2022$ The Author(s). Published by Wolters Kluwer Health, Inc. on behalf of the American

Academy of Neurology.. All rights reserved. Online ISSN: 2332-7812.

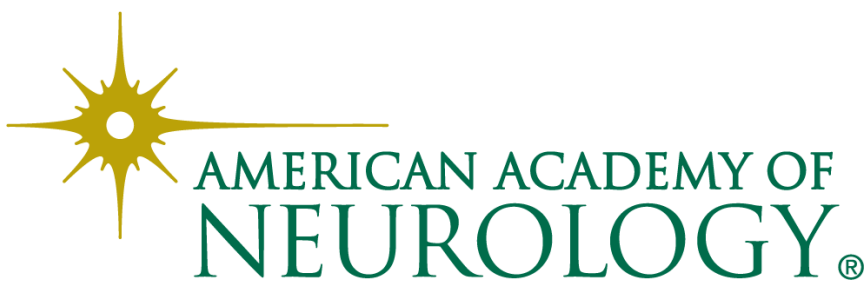

